\title{
Integration of peroxisomes into an endomembrane system that governs cellular aging
}

\author{
Adam Beach ${ }^{\dagger}$, Michelle T. Burstein ${ }^{\dagger}$, Vincent R. Richard ${ }^{\dagger}$, Anna Leonov $^{\dagger}$, Sean Levy ${ }^{\dagger}$ and \\ Vladimir I. Titorenko*
}

Department of Biology, Concordia University, Montreal, PQ, Canada

\section{Edited by:}

Troy Harkness, University of

Saskatchewan, Canada

Reviewed by:

Yasser M. EL-WAZIR, Suez Canal

University, Egypt

Troy Harkness, University of

Saskatchewan, Canada

*Correspondence:

Vladimir I. Titorenko, Department of

Biology, Concordia University, 7141

Sherbrooke Street, West, Montreal,

$P Q, H 4 B$ 1R6, Canada.

e-mail:vtitor@alcor.concordia.ca

tThese authors contributed equally to this work.
The peroxisome is an organelle that has long been known for its essential roles in oxidation of fatty acids, maintenance of reactive oxygen species (ROS) homeostasis and anaplerotic replenishment of tricarboxylic acid (TCA) cycle intermediates destined for mitochondria. Growing evidence supports the view that these peroxisome-confined metabolic processes play an essential role in defining the replicative and chronological age of a eukaryotic cell. Much progress has recently been made in defining molecular mechanisms that link cellular aging to fatty acid oxidation, ROS turnover, and anaplerotic metabolism in peroxisomes. Emergent studies have revealed that these organelles not only house longevity-defining metabolic reactions but can also regulate cellular aging via their dynamic communication with other cellular compartments. Peroxisomes communicate with other organelles by establishing extensive physical contact with lipid bodies, maintaining an endoplasmic reticulum (ER) to peroxisome connectivity system, exchanging certain metabolites, and being involved in the bidirectional flow of some of their protein and lipid constituents. The scope of this review is to summarize the evidence that peroxisomes are dynamically integrated into an endomembrane system that governs cellular aging. We discuss recent progress in understanding how communications between peroxisomes and other cellular compartments within this system influence the development of a pro- or anti-aging cellular pattern. We also propose a model for the integration of peroxisomes into the endomembrane system governing cellular aging and critically evaluate several molecular mechanisms underlying such integration.

Keywords: peroxisome, cellular aging, interorganellar communication, sirtuins, senescence factors, organelle inheritance, proteostasis, autophagy

\section{INTRODUCTION}

A growing body of evidence implies that, in addition to the well known roles of the peroxisome in housing fatty acid oxidation and maintaining hydrogen peroxide homeostasis (Poirier et al., 2006; Wanders and Waterham, 2006; Schlüter et al., 2010), this organelle is actively involved in organizing the processes of development, differentiation, and morphogenesis in evolutionarily distant organisms. In mammalian and plant cells, the rate of fatty acid metabolism and the efficiency of reactive oxygen species (ROS) and reactive nitrogen species (RNS) turnover within the peroxisome define the dynamics of changes in the levels of signaling lipids, ROS, and RNS outside this organelle (Desvergne and Wahli, 1999; Corpas et al., 2001; del Río et al., 2006; Nyathi and Baker, 2006). Following their release from the peroxisome, these signaling molecules bind and activate a distinct set of transcription factors that respond by causing global changes in gene expression to initiate certain developmental and differentiation programs (Kersten et al., 2000; Desikan et al., 2001; Hu et al., 2002; Ma et al., 2002; Michalik et al., 2002; Baker et al., 2006; del Río et al., 2006; Michalik and Wahli, 2006; Nyathi and Baker, 2006; Bonekamp et al., 2009; Antonenkov et al., 2010; Ivashchenko et al., 2011; Li et al., 2011; Neher et al., 2012). Thus, the peroxisome functions as an intracellular signaling compartment that can orchestrate important developmental decisions from inside the cell by modulating the extra-peroxisomal concentrations of several potent cellular messengers (Titorenko and Rachubinski, 2004; Terlecky and Titorenko, 2009; Thoms et al., 2009; Dixit et al., 2010). Furthermore, the peroxisome can operate as an organizing platform for several developmental and differentiation programs by compartmentalizing the initial steps of plasmalogen biosynthesis in mammalian and nematode cells, providing acetyl-CoA for the biosynthesis of melanin and glycerol in fungal cells, and carrying out the oxidative decomposition of very long-chain fatty acids, phytanic acid, and pristanic acid in mammalian cells (Powers and Moser, 1998; Motley et al., 2000; Thines et al., 2000; Gould et al., 2001; Kimura et al., 2001; Petriv et al., 2002; Wang et al., 2005; Asakura et al., 2006; Terlecky and Titorenko, 2009; Imazaki et al., 2010; Van Veldhoven, 2010; Goh et al., 2011; Mast et al., 2011; Bhadauria et al., 2012). Moreover, while the peroxisome-associated pools of several bifunctional proteins with dual subcellular localization operate in peroxisome biogenesis and function, their pools in other organelles organize certain processes of development, differentiation, and morphogenesis in mammalian, plant, and yeast cells (Titorenko et al., 1997; Titorenko and Rachubinski, 1998, 2004; Lin et al., 1999; 
Footitt et al., 2002; Gavva et al., 2002; Geuze et al., 2003; Lin et al., 2004; Slabas et al., 2004; Karnik and Trelease, 2005; Ashibe et al., 2007; Freitag et al., 2012). In addition, the peroxisome provides a template for the formation of the Woronin body, a specialized subcellular compartment that in the filamentous fungi Neurospora crassa and Aspergillus oryzae is essential for a multistep process in cell morphogenesis initiated by physical damage to hyphae (Jedd and Chua, 2000; Tenney et al., 2000; Liu et al., 2008; Escaño et al., 2009; Jedd, 2011; Liu et al., 2011). In human cells, the peroxisome can also serve as an intracellular platform for the development of the human immunodeficiency virus and rotavirus (Cohen et al., 2000; Mohan et al., 2002).

Recent findings have broadened a spectrum of complex biological processes that depend on the functional integrity of the peroxisome. Emergent evidence supports the view that such peroxisome-confined metabolic processes as fatty acid oxidation, ROS turnover, and anaplerotic replenishment of tricarboxylic acid (TCA) cycle intermediates play essential roles in defining the replicative and chronological age of a eukaryotic cell (Titorenko and Terlecky, 2011). Peroxisomal fatty acid oxidation has been shown to regulate cellular aging because it operates as a system controller that modulates levels of non-esterified fatty acids and diacylglycerol by governing lipid dynamics in peroxisomes, lipid bodies, and the endoplasmic reticulum (ER) (Goldberg et al., 2009a,b; Titorenko and Terlecky, 2011); non-esterified fatty acids are known to accelerate the age-related necrotic and apoptotic cell death mechanisms, whereas the diacylglycerol-activated protein kinase $\mathrm{C}$ signaling sensitizes cells to age-related stresses (Spitaler and Cantrell, 2004; Low et al., 2005; Feng et al., 2007; Aksam et al., 2008; Jungwirth et al., 2008). Furthermore, peroxisomal fatty acid oxidation and anaplerotic reactions have been demonstrated to delay cellular aging by potentiating the mitochondrial retrograde (RTG) signaling pathway of longevity regulation (Chelstowska and Butow, 1995; Kos et al., 1995; Epstein et al., 2001; Traven et al., 2001; Jazwinski, 2005b; Liu and Butow, 2006; Titorenko and Terlecky, 2011; Jazwinski, 2012). Moreover, ROS homeostasis and the extent of macromolecular oxidative damage within the peroxisome govern several anti-aging processes confined to this organelle (Morita et al., 2000; Legakis et al., 2002; Aksam et al., 2007; Koepke et al., 2008; Aksam et al., 2009; Lingard et al., 2009; Mathur, 2009; Sinclair et al., 2009; Titorenko and Terlecky, 2011).

The peroxisome defines the replicative and chronological age of a eukaryotic cell not only by operating as a system controller that modulates levels of non-esterified fatty acids and diacylglycerol, replenishes TCA cycle intermediates destined for mitochondria, and contributes to the maintenance of peroxisomal ROS homeostasis and macromolecular oxidative damage. Recent studies have revealed that this organelle can also regulate cellular aging via its communication with other cellular compartments. This dynamic communication involves the establishment of extensive physical contact between peroxisomes and lipid bodies, maintenance of an ER to peroxisome connectivity system, exchange of certain metabolites between peroxisomes and other cellular compartments, and bidirectional flow of some protein and lipid constituents between peroxisomes and other organelles. In this review we summarize the evidence that peroxisomes are dynamically integrated into an endomembrane system that governs cellular aging. We discuss various strategies through which peroxisomes are integrated into this endomembrane system, critically evaluate the molecular mechanisms underlying each of these strategies, and analyze the age-related dynamics of communications between peroxisomes and other cellular compartments composing the longevity-defining endomembrane system. We also outline recent progress in understanding how communications between peroxisomes and other cellular compartments within this system influence the development of a pro- or antiaging cellular pattern. Based on the available evidence, we propose a model for the integration of peroxisomes into the endomembrane system governing cellular aging.

\section{A ROLE FOR CYTOSOL-TO-PEROXISOME TARGETING OF Pnc1p IN REGULATING YEAST LONGEVITY}

A support for a distinctive mechanism that underlies the essential role of peroxisomes in regulating cellular aging comes from the observation that Pnclp, a pyrazinamidase/nicotinamidase 1 that converts nicotinamide to nicotinic acid in the $\mathrm{NAD}^{+}$ salvage pathway (Ghislain et al., 2002), is targeted from the cytosol to the peroxisome in response to $\mathrm{CR}$ and various mild stresses (Anderson et al., 2003). CR and all of these other "hormetic" stimuli-the term "hormesis" refers to a beneficial defense response of an organism to a low-intensity biological stress (Gems and Partridge, 2008; Rattan, 2008; Calabrese et al., 2011, 2012) - increase the lifespan of replicatively aging yeast in a Pnclp-dependent manner (Anderson et al., 2003). Peroxisomal import of Pnclp under conditions of such longevity-extending hormesis requires the peroxisomal targeting signal 2 (PTS2) shuttling receptor Pex7p and the peroxin Pex6p, but does not rely on the PTS1 receptor Pex5p (Anderson et al., 2003). Such specific peroxisomal targeting of Pnclp, one of the key regulators of replicative aging in yeast (Lin and Sinclair, 2008), in response to their exposure to various anti-aging exogenous factors suggests that Pnclp in the peroxisome could modulate some longevity-related processes confined to this organelle. What are these processes?

The established function of Pnclp in the nucleus-an organelle to which this protein is also sorted from the cytosol in yeast exposed to CR and other hormetic stimuli (Anderson et al., 2003) — provides a useful hint on the nature of peroxisomeconfined processes that could be modulated by Pnclp under these longevity-extending conditions. In the nucleus, Pnclp depletes the level of nicotinamide, a strong non-competitive inhibitor of the $\mathrm{NAD}^{+}$-dependent protein deacetylase Sir2p required for lifespan extension in yeast under CR conditions (Bitterman et al., 2002). The resulting Pnclp-driven activation of Sir2p delays replicative aging by suppressing recombination at the ribosomal DNA (rDNA) locus, thereby decreasing the efficiency of extrachromosomal rDNA circle (ERC) formation in the nucleolus (Lin and Sinclair, 2008). It should be stressed that two of the four members of the Sir2p family of $\mathrm{NAD}^{+}$-dependent protein deacetylases (i.e., sirtuins) in yeast—called Hst3p and Hst4p for being Homologs of SIR Two proteins-drive the metabolism of fatty acids by activating acyl-CoA synthetases for their short-chain species (Starai et al., 2003). By converting 
short-chain fatty acids into their corresponding acyl-CoA forms, acyl-CoA synthetases enable their cellular and intracellular transport and metabolism (Starai et al., 2003). It has been proposed that both Hst3p and Hst4p activate these acyl-CoA synthetases by deacetylating them and cleaving $\mathrm{NAD}^{+}$in each reaction cycle (Starai et al., 2003). Because sirtuins are also known for their $\mathrm{NAD}^{+}$-dependent ADP-ribosylation activity (Haigis and Guarente, 2006; Haigis and Sinclair, 2010), a possibility that Hst3p and Hst4p activate acyl-CoA synthetases for shortchain fatty acids in ADP-ribosylation reactions is also feasible. Altogether, these findings suggest the following hypothesis for a role of cytosol-to-peroxisome targeting of Pnclp in regulating longevity of replicatively aging yeast (Figure 1). In response to their exposure to CR and other hormetic anti-aging stimuli, yeast cells target Pnclp not only to the nucleus but also to the peroxisome. Following its PTS2- and Pex7p-dependent import into the peroxisome, Pnclp depletes the level of nicotinamide, a strong non-competitive inhibitor of Hst3p and Hst4p. As a co-substrate in protein deacetylation and/or ADP-ribosylation reactions, each of these sirtuins could use $\mathrm{NAD}^{+}$known to be generated by the peroxisomal malate dehydrogenase Mdh3p (Kunze et al., 2006). The Pnclp-dependent depletion of nicotinamide activates Hst3p and Hst4p; in turn, these sirtuins stimulate acyl-CoA synthetases required for peroxisomal transport and oxidation of short-chain fatty acids (Figure 1). We hypothesize that, by depleting the levels of these fatty acids in the cytosol and/or oxidizing them, peroxisomes make an important contribution to the longevity-extending effect of CR and other

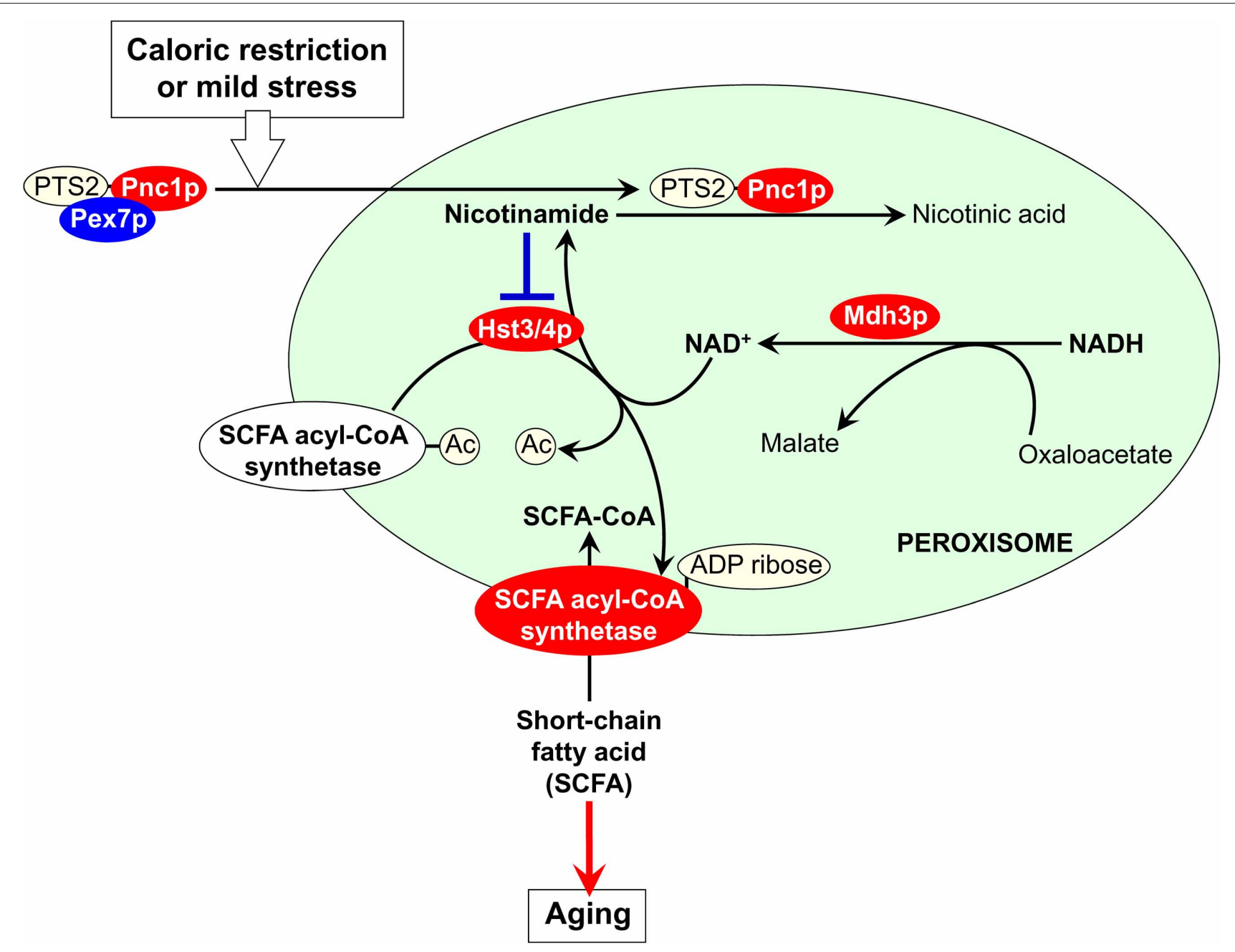

FIGURE 1 | A proposed role for cytosol-to-peroxisome targeting of Pnc1p in regulating longevity of replicatively aging yeast. If exposed to caloric restriction (CR) and other hormetic anti-aging stimuli, yeast cells respond by targeting Pnc1p - a pyrazinamidase/nicotinamidase 1 that converts nicotinamide to nicotinic acid in the $\mathrm{NAD}^{+}$salvage pathway-not only to the nucleus but also to the peroxisome. The delivery of Pnc1p to the peroxisome depends on the peroxisomal targeting signal 2 (PTS2) shuttling receptor Pex7p. Inside the peroxisome, Pnc1p activates the sirtuins Hst3p and $\mathrm{Hst} 4 \mathrm{p}$ by reducing the concentration of their non-competitive inhibitor nicotinamide. Using $\mathrm{NAD}^{+}$generated by the peroxisomal malate dehydrogenase Mdh3p as a co-substrate in protein deacetylation and ADP-ribosylation reactions, the activated Hst3p and Hst4p stimulate acyl-CoA synthetases required for peroxisomal transport and oxidation of short-chain fatty acids (SCFA). By reducing the levels of these fatty acids in the cytosol, peroxisomes contribute to the beneficial effect of CR and other hormetic stimuli on longevity. See text for details. 
hormetic stimuli. A critical evaluation of our hypothesis will require testing of the localization of Hst3p and Hst4p to the peroxisome, either permanent or triggered in response to CR and mild stresses. Another key challenge for the future will be to evaluate the ability of peroxisomal acyl-CoA synthetases to undergo reversible deacetylation and/or ADP-ribosylation in an Hst3pand/or Hst4p-dependent fashion following exposure of yeast to these longevity-extending stimuli.

\section{THE PEROXIN Pex6p CONTRIBUTES TO THE MAINTENANCE OF AGE ASYMMETRY BETWEEN THE MOTHER AND DAUGHTER YEAST CELLS WITH RESPECT TO SEGREGATION OF FUNCTIONAL MITOCHONDRIA}

Several peroxisomal proteins are known to possess dual subcellular localization and function (reviewed by Titorenko and Rachubinski, 2004; Mast et al., 2010; Islinger et al., 2012). While the major, peroxisome-bound portion of each of these proteins controls essential processes confined to this organelle, their pools in other organellar compartments govern certain developmental, differentiation, and morphogenetic programs (Titorenko and Rachubinski, 2004; Islinger et al., 2012). The emerged compendium of these bifunctional peroxisomal proteins with dual subcellular localization is on a fast-growing list of the so-called "moonlighting proteins" (Jeffery, 1999, 2011; Shi and Shi, 2004; Kim and Dang, 2005; Gancedo and Flores, 2008; Jeffery, 2009; Flores and Gancedo, 2011). By analyzing the information on dynamic changes in metabolic status and/or organelle functional state within one subcellular location and then moving to other location(s) for initiating an adequate response to such changes, these moonlighting proteins integrate various cellular activities in space and time (Shi and Shi, 2004; Kim and Dang, 2005; Cho et al., 2006; Gancedo and Flores, 2008; Sen et al., 2008; Flores and Gancedo, 2011; Jeffery, 2011).

The peroxin Pex6p is an AAA ATPase (ATPase associated with various cellular activities) whose peroxisome-associated pool has been implicated in peroxisomal protein import (Titorenko and Rachubinski, 2009; Ma et al., 2011; Rucktäschel et al., 2011). In the yeast Yarrowia lipolytica Pex6p is a moonlighting protein whose minor portion is confined to the ER (Titorenko et al., 1997; Titorenko and Rachubinski, 1998). The ER-associated Pex6p is an essential component of protein machinery that orchestrates the dimorphic transition from a round yeast form to a filamentous (mycelial) form (Titorenko et al., 1997; Titorenko and Rachubinski, 2004). Pex6p, along with other ER components of this machinery, governs this cell polarization and differentiation program by driving the delivery of mycelium-specific proteins from the ER to the cell surface (Titorenko et al., 1997).

Recently, a list of the "extra-curricular" activities of Pex6p has been updated by including to it the essential role that this peroxin plays in regulating yeast longevity. Because the yeast Saccharomyces cerevisiae reproduce by asymmetric cell division, replicatively "young" mother cells retain such "senescence factors" (also called "aging factors") as ERCs, oxidatively damaged proteins, protein aggregates, and dysfunctional mitochondria (Figure 2A) (Jazwinski, 2005a; Erjavec et al., 2007, 2008; Henderson and Gottschling, 2008; Steinkraus et al., 2008; Eldakak et al., 2010; Liu et al., 2010; Zhou et al., 2011).
Their budding progeny therefore retains the full replicative capacity by not inheriting ERCs or damaged/aggregated proteins and receiving only functional mitochondria (Henderson and Gottschling, 2008; Erjavec et al., 2008; Steinkraus et al., 2008). In replicatively "old" mother cells, this age asymmetry between the mother and daughter cells is lost. As a result, the daughters inherit all four of the known senescence factors (Figure 2A) (Jazwinski, 2005a; Henderson and Gottschling, 2008; Steinkraus et al., 2008). It should be stressed that the overexpression of Pex6p suppresses the lack of age asymmetry between mother and daughter cells in a strain carrying a point mutation in the nuclear gene ATP2 encoding the $\beta$-subunit of the $\mathrm{F}_{1}$ sector of mitochondrial $\mathrm{F}_{0}, \mathrm{~F}_{1}$ ATP synthase (Lai et al., 2002; Seo et al., 2007). Moreover, not only Pex6p-along with yet-to-be-identified cytosolic proteinsfacilitates the import of Atp2p into mitochondria, but it also drives the segregation of functional mitochondria to daughter cells (Seo et al., 2007). Therefore, it is conceivable that Pex6p could operate as one of the "filters" sequestering dysfunctional mitochondria in the mother cell and/or segregating functional mitochondria to the daughter cell (Figures 2B and 2C). The challenge remains to define the mechanisms underlying the ability of Pex6 to facilitate mitochondrial import of Atp2p and to maintain the age-related asymmetrical segregation of functional mitochondria between mother and daughter cells. Another key challenge for the future will be to establish the mechanism for delivery of Pex6 from peroxisomes to mitochondria. Importantly, not only the biogenesis of these two organelles is governed by common transcriptional pathways, but they also share several key components of their division machineries and are linked through mitochondria-to-peroxisome vesicular traffic (Figures 2B and 2C) (Liu and Butow, 2006; Neuspiel et al., 2008; Andrade-Navarro et al., 2009; Delille et al., 2009; Jazwinski, 2012; Islinger et al., 2012).

\section{TWO MECHANISMS FOR PREVENTING THE SEGREGATION OF DYSFUNCTIONAL, OXIDATIVELY DAMAGED PEROXISOMES TO THE DAUGHTER YEAST CELL DURING MITOSIS}

Not only peroxisomes in replicatively aging yeast contribute to selective segregation of functional mitochondria to the daughter cell, but they also possess a protein machine that governs their own distribution between mother and daughter cells. Recent studies suggested two mechanisms by which this protein machine may operate in preventing the inheritance of dysfunctional, oxidatively damaged peroxisomes by the daughter cell during mitosis.

In $S$. cerevisiae, the inheritance of peroxisomes by daughter cells relies on the peroxisomal protein Inp2p (Fagarasanu et al., 2006). By acting as a receptor for the class $\mathrm{V}$ myosin motor Myo2p, Inp2p tags peroxisomes for their segregation to the daughter cell (Fagarasanu et al., 2009). It is conceivable that such Inp2p-dependent tagging of peroxisomes plays a longevity-extending role by enabling the inheritance of only functional peroxisomes by daughter cells. Importantly, the phosphorylation of Inp $2 \mathrm{p}$ makes it susceptible to degradation, thereby impairing the segregation of Inp2p-less peroxisomes to the daughter cell (Fagarasanu et al., 2009, 2010). One could therefore 


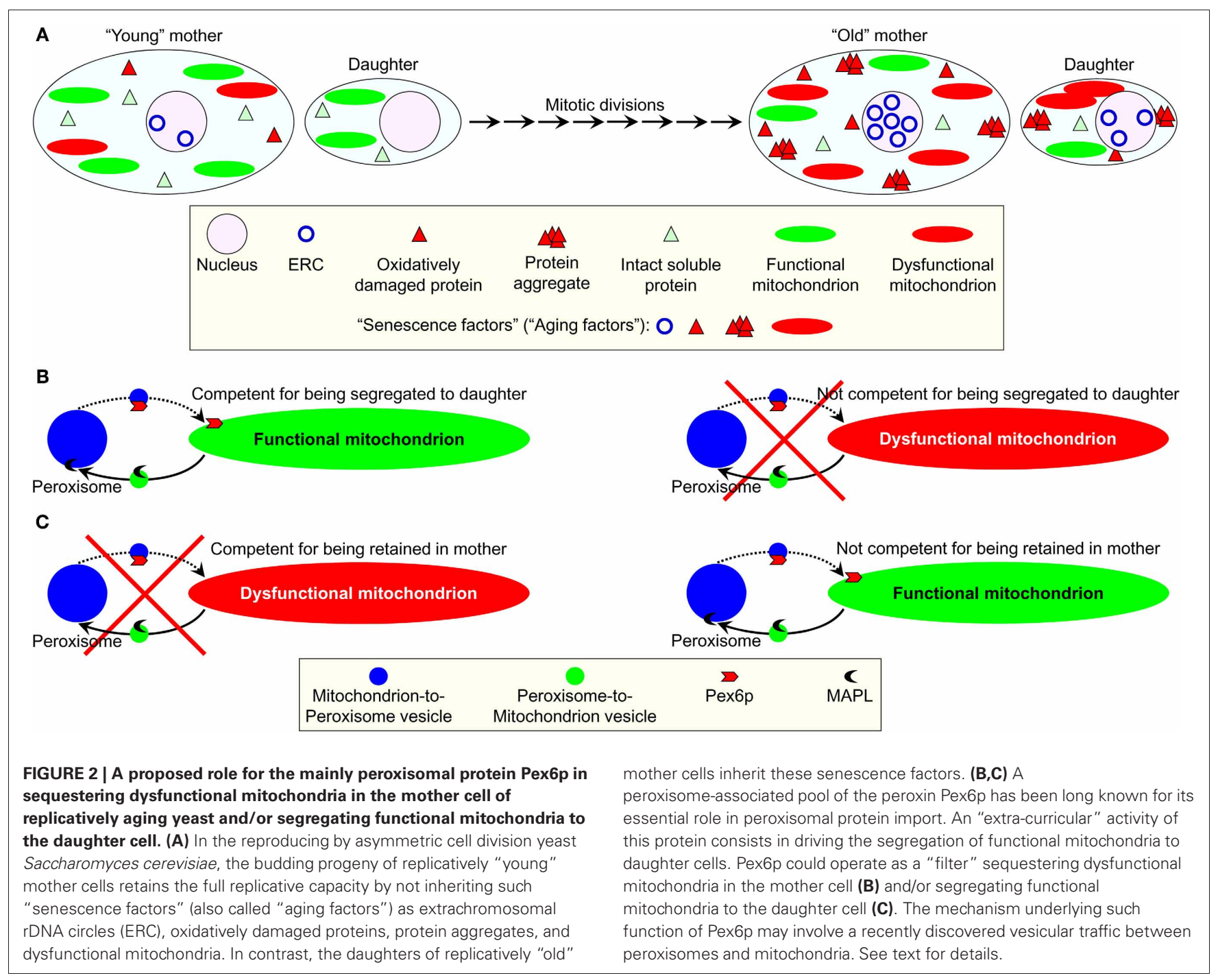

speculate that such phosphorylation and degradation target mainly Inp2p on dysfunctional, oxidatively damaged peroxisomes for sequestering them in the mother cell.

In another yeast species, Y. lipolytica, the inheritance of only newly formed from the ER template peroxisomes may prevent the segregation of their oxidatively damaged, "old" counterparts to the daughter cell during mitosis (Chang et al., 2009). By possessing a dual role in the formation of new peroxisomes from the ER template and in the recruitment of the class $\mathrm{V}$ myosin motor Myo2p to their membranes, the peroxins Pex3p and Pex3Bp may enable the selective segregation of these newly formed peroxisomes to the daughter cell, thereby allowing to retain the entire population of dysfunctional, oxidatively damaged peroxisomes in the mother cell (Chang et al., 2009; Fagarasanu et al., 2010).

The challenge remains to define the molecular mechanisms underlying the proposed selectivity in (1) phosphorylating Inp2p only on dysfunctional, oxidatively damaged peroxisomes; and (2) targeting Myo2p only to the ER-confined pool of Pex3p.

\section{A MODEL FOR THE INTEGRATION OF PEROXISOMES INTO AN ENDOMEMBRANE SYSTEM THAT GOVERNS CELLULAR AGING}

A body of evidence summarized here and elsewhere (Titorenko and Rachubinski, 2004; Titorenko and Terlecky, 2011; Islinger et al., 2012) implies that peroxisomes contribute to the regulation of cellular aging via several different mechanisms. In each of these mechanisms, peroxisomes communicate with other organelles by establishing extensive physical contact with lipid bodies, maintaining the ER-peroxisome connectivity, exchanging certain metabolites, and/or being involved in the bidirectional flow of some of their protein and lipid constituents. Thus, peroxisomes are dynamically integrated into an endomembrane system that governs cellular aging. We propose a model for such integration (Figure 3). The central tenet of this model is that the age-dependent efficiency of protein import into the peroxisome modulates the dynamics of its communication with other cellular compartments, thereby influencing several longevity regulation pathways that rely on such communication. The overall efficiency of peroxisomal protein import is defined by the efficiencies 


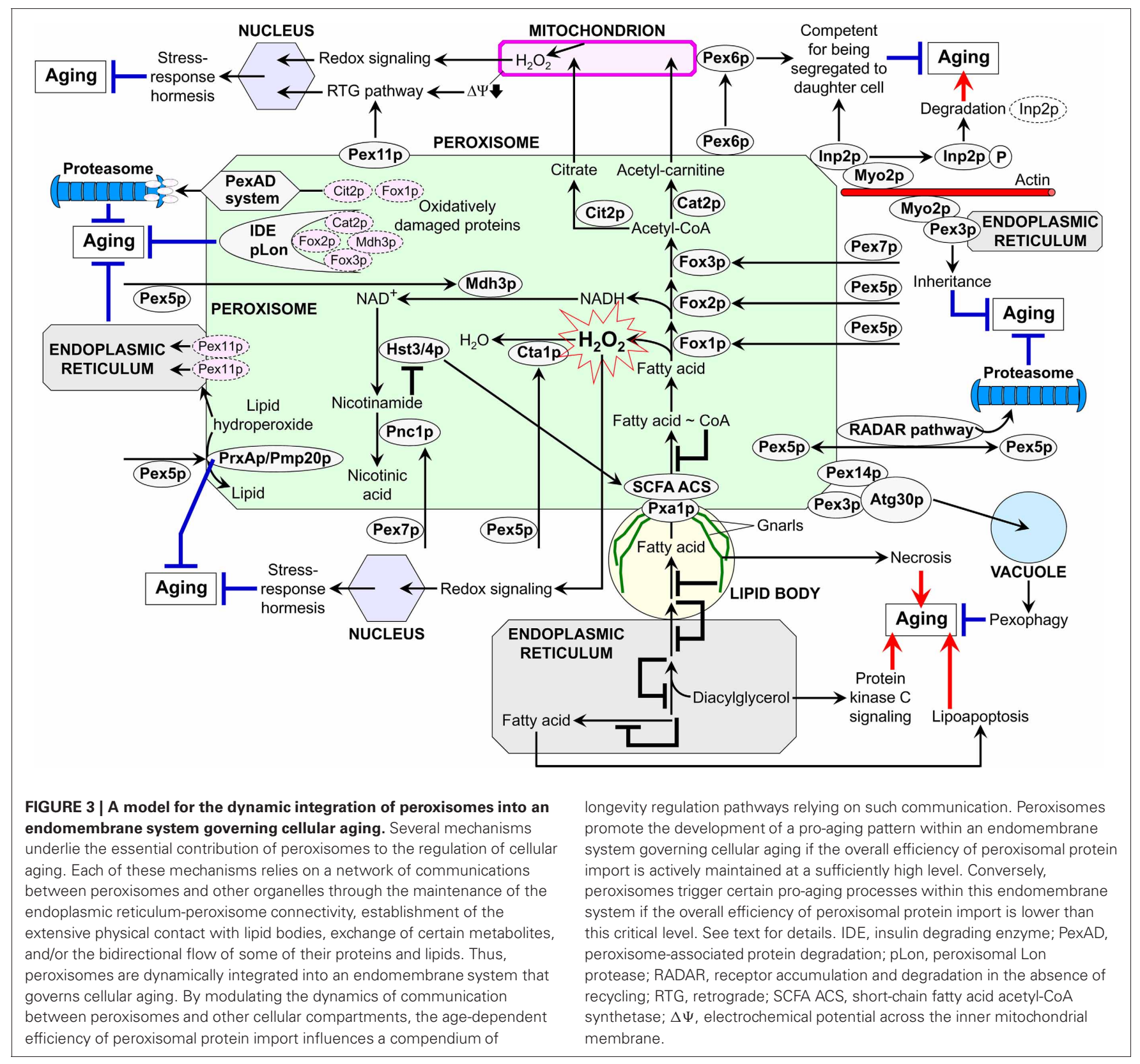

of binding of Pex5p and Pex7p-the PTS1 and PTS2 cytosolic shuttling receptors, respectively-to their cargo proteins in the cytosol, translocation of the receptor-cargo complexes across the peroxisomal membrane, and receptor recycling (Ma et al., 2011; Rucktäschel et al., 2011). Importantly, the efficiencies of all these processes are reduced with age (Legakis et al., 2002; Terlecky et al., 2006; Titorenko and Terlecky, 2011). In our model, if the overall efficiency of protein import into peroxisomes is actively maintained at a sufficiently high level, these organelles trigger certain anti-aging processes within the endomembrane system governing cellular aging (Figure 3). Conversely, if the overall efficiency of peroxisomal protein import is lower than this critical level, peroxisomes promote the development of a pro-aging pattern within this endomembrane system (Figure 3).
Our model envisions that the efficient Pex5p-dependent peroxisomal import of the ROS scavenging enzymes catalase (Ctalp in yeast) and peroxiredoxin (PrxAp in mammals and Pmp20p in yeast) in replicatively and chronologically "young" cells aids in minimizing the oxidative damage to peroxisomal proteins and membrane lipids (Figure 3; Antonenkov et al., 2010; Titorenko and Terlecky, 2011; Ivashchenko et al., 2011). At the surface of the peroxisome, quality control of the Pex $5 p$-driven protein import in these cells is governed by the receptor accumulation and degradation in the absence of recycling (RADAR) pathway for the recycling of Pex5p (Figure 3; Léon et al., 2006; Ma et al., 2011; Titorenko and Terlecky, 2011). Inside the peroxisome, the insulin degrading enzyme (IDE), peroxisomal Lon (pLon) protease, and peroxisome-associated protein degradation (PexAD) system carry 
out the degradation of oxidatively damaged peroxisomal matrix proteins that are incapable of supporting the anti-aging processes orchestrated by functionally active peroxisomes (Morita et al., 2000; Aksam et al., 2007, 2009; Lingard et al., 2009). A healthy population of such peroxisomes in "young" cells is also sustained by pexophagy, an autophagy-related process in which dysfunctional peroxisomes carrying oxidatively damaged proteins are selectively degraded following their sequestration by vacuoles of yeast and plant cells or lysosomes of mammalian cells (Figure 3; Aksam et al., 2007; Farré et al., 2008; Manjithaya et al., 2010). In plant cells exposed to oxidative stress, the ER-peroxisome connectivity enables the retro-flow of oxidatively damaged matrix proteins as well as of membrane proteins and lipids to the ER-thereby contributing to the maintenance of a healthy population of functionally active peroxisomes (Mathur, 2009; Sinclair et al., 2009).

In our model, peroxisomes in "young" cells not only actively avoid the oxidative damage to their protein and lipid constituents but also operate as a signaling platform that, by maintaining ROS concentration at a certain "optimal" level, delays cellular aging by inducing stress-response hormesis (Figure 3; Titorenko and Terlecky, 2011). At such a level, ROS are unable to elicit substantial oxidative damage to cellular macromolecules but can activate several redox signaling networks known to elevate the abundance and/or activity of stress-protecting and other anti-aging proteins (D'Autréaux and Toledano, 2007; Giorgio et al., 2007; Veal et al., 2007).

Furthermore, the PTS1 and PTS2 cytosolic shuttling receptors Pex5p and Pex7p drive peroxisomal import of Fox1p, Fox 2p, and Fox3p (Hiltunen et al., 2003). The efficient import of these core enzymes of fatty acid $\beta$-oxidation into peroxisomes of "young" cells increases the efficacy with which they decompose fatty acids derived from triacylglycerols that are synthesized in the ER and deposited within lipid bodies (Goodman, 2008; Goldberg et al., 2009a,b; Kohlwein, 2010). Due to such accelerated peroxisomal fatty acid oxidation and the resulting decrease in the concentrations of non-esterified fatty acids and diacylglycerol, "young" cells escape the premature death by resisting lipid-induced necrosis and apoptosis and by sustaining stress resistance through the attenuation of diacylglycerol-activated protein kinase $\mathrm{C}$ signaling (Figure 3; Goldberg et al., 2009a,b; Titorenko and Terlecky, 2011). Another way for the longevity-extending acceleration of peroxisomal fatty acid oxidation in "young" cells is the governed by sirtuins Hst3p and Hst4p stimulation of acyl-CoA synthetases that are required for peroxisomal transport and oxidation of short-chain fatty acids. This anti-aging process is driven by the efficient Pex5p- and Pex7p-dependent peroxisomal import of Mdh3p and Pnclp for synthesizing a substrate and decomposing an inhibitor of the sirtuins, respectively (Figure 3).

Moreover, the longevity-extending ability of peroxisomes to promote the anti-aging RTG signaling pathway of peroxisomesmitochondria, mitochondria-nucleus, and nucleus-peroxisomes communications in "young" cells is enhanced by the highly efficient peroxisomal import of Fox1p, Fox $2 p$, Fox $3 p$, Cit2p, and Cat $2 \mathrm{p}$ in these cells (Figure 3; Titorenko and Terlecky, 2011). Fox 1 , Fox $2 p$, and Fox $3 p$ are involved in the peroxisomal oxidation of fatty acid to acetyl-CoA following their
Pex5p- and Pex7p-dependent delivery to peroxisomes, whereas the citrate synthase Cit2p and acetyl-carnitine synthase Cat2p are imported into these organelles with the help of Pex5p to catalyze the anaplerotic conversion of acetyl-CoA to citrate and acetyl-carnitine (Figure 3; Epstein et al., 2001; Traven et al., 2001; Hiltunen et al., 2003; Titorenko and Terlecky, 2011). The longevity-extending RTG signaling pathway in "young" cells is further amplified through the Pex11p-driven proliferation of peroxisomes and the resulting increase in the effectiveness with which the confined to these organelles fatty acid oxidation and anaplerotic reactions replenish TCA cycle intermediates destined for mitochondria (Figure 3; Jazwinski, 2005b; Liu and Butow, 2006; Titorenko and Terlecky, 2011). It should be emphasized that, by maintaining the functionality of mitochondria in "young" cells, the peroxisome-driven RTG pathway controls the homeostasis of mitochondrial ROS (Titorenko and Terlecky, 2011). This enables the ROS-dependent activation of several redox signaling networks aimed at increasing the levels of stressprotecting and other anti-aging proteins or post-translationally activating some of them (Figure 3; D'Autréaux and Toledano, 2007; Giorgio et al., 2007; Veal et al., 2007). In our model, the segregation of functional mitochondria to the "young" daughter cell and/or the sequestration of dysfunctional mitochondria in the "old" mother cell in replicatively aging yeast are/is driven in part by the delivery of the peroxin Pex6 from peroxisomes to mitochondria through a mechanism that remains to be established (Figure 3; Lai et al., 2002; Seo et al., 2007).

According to our model, $S$. cerevisiae Inp2p-a peroxisomespecific receptor for the class $\mathrm{V}$ myosin motor Myo2p-tags peroxisomes for their segregation to the "young" daughter cell in a process that may play a life-extending role by enabling the inheritance of only functional peroxisomes (Figure 3; Fagarasanu et al., 2009, 2010). Furthermore, by possessing a dual role in the formation of new peroxisomes from the ER template and in the recruitment of Myo2p to their membranes, the Y. lipolytica peroxins Pex3p and Pex3Bp may enable the inheritance of only newly formed from the ER template peroxisomes thus preventing the segregation of their oxidatively damaged, "old" counterparts to the daughter cell during mitosis (Figure 3; Chang et al., 2009; Fagarasanu et al., 2010).

Our model envisions that the overall efficiency of peroxisomal protein import gradually decreases with replicative and chronological age (Figure 3). A steady, age-related increase in the concentration of peroxisome-confined proteins that are oxidatively damaged by peroxisomally produced ROS could be the driving force for such deterioration of peroxisomal protein import efficiency. The Pex5p-dependent peroxisomal import of catalasedue to the age-dependent decline in the efficiency of its binding to Pex $5 p$ and in the extent of Pex5p recycling-is the most sensitive to oxidative damage peroxisomal process (Legakis et al., 2002; Terlecky et al., 2006). The resulting deceleration of catalase import into peroxisomes increases the extent of oxidative damage to their proteins and lipids, thereby initiating the "deterioration spiral" that eventually lowers the overall efficiency of peroxisomal protein import below a critical level. Consequently, the role of peroxisomes in the regulation of cellular aging is switching from being a platform for activating a compendium of 
anti-aging processes within the endomembrane system governing cellular aging to becoming a platform for the development of a pro-aging pattern within this endomembrane system (Titorenko and Terlecky, 2011). Specifically, the RADAR pathway, IDE and pLon proteases, PexAD system, and pexophagy eventually fail due to the progressive, age-dependent accumulation of oxidatively damaged proteins and lipids in peroxisomes. Thus, cellular aging coincides with the build-up of dysfunctional peroxisomes that are unable anymore to support the anti-aging processes within the endomembrane system governing such aging. Among these impaired anti-aging processes are (1) the peroxisomeand mitochondria-driven pathways of stress response hormesis; (2) the Hst3p/Hst4p-dependent stimulation of acyl-CoA synthetases for peroxisomal transport and oxidation of short-chain fatty acids; (3) the RTG signaling pathway of peroxisomesmitochondria, mitochondria-nucleus, and nucleus-peroxisomes communications; (4) the Pex6p-dependent sequestration of dysfunctional mitochondria in the "old" mother cell and/or segregation of functional mitochondria to the "young" daughter cell; and (5) the Inp2p-, Pex3p-, and Pex3Bp-dependent segregation of functional peroxisomes to the "young" daughter cell (Figure 3). Moreover, our model foresees that, by being unable to maintain low levels of non-esterified fatty acids and diacylglycerol, the dysfunctional, oxidatively damaged peroxisomes accumulated in aged cells (1) activate the longevity-shortening necrotic and apoptotic cell death mechanisms induced by non-esterified fatty acids; and (2) are unable to attenuate the diacylglycerolactivated protein kinase $\mathrm{C}$ signaling that reduces stress resistance (Figure 3).

\section{CONCLUSION}

Growing evidence supports the view that peroxisomes govern cellular aging via several different mechanisms involving their dynamic communication with other cellular compartments. An important conceptual advance in our understanding of the inherent complexity of cellular aging is that the age-related dynamics of communications between peroxisomes and various other organelles modulates a compendium of longevity regulation pathways. It is conceivable therefore that the peroxisome is dynamically integrated into an endomembrane system governing cellular aging. Much progress has recently been made in defining how communications between peroxisomes and other cellular compartments influence the development of a pro- or antiaging pattern within this endomembrane system. The challenge remains to define the molecular mechanisms underlying the integration of peroxisomes into the endomembrane system governing cellular aging. Future work will aim at understanding how peroxisomes switch their role in the regulation of cellular aging from being a platform for activating a compendium of anti-aging processes confined to this endomembrane system in "young" cells to becoming a platform for the development of a pro-aging pattern within this endomembrane system in "old" cells. This knowledge will provide greater insight into the mechanisms underlying longevity regulation and is expected to reveal novel targets for anti-aging pharmaceuticals that can extend longevity by modulating the age-related dynamics of communications between peroxisomes and other cellular compartments.

\section{ACKNOWLEDGMENTS}

Vladimir I. Titorenko research is supported by grants from the NSERC of Canada and Concordia University Chair Fund. P.K. was supported by Doctoral Research Fellowship Awards from the Fonds de recherché en santé du Quebec and from the Fonds québécois de la recherche sur la nature et les technologies (FQRNT). Adam Beach and Vincent R. Richard were supported by Frederick Banting and Charles Best Doctoral Scholarship Awards from the Canadian Institutes of Health Research. Michelle T. Burstein was supported by a Doctoral Research Fellowship Award from the FQRNT. Vladimir I. Titorenko is a Concordia University Research Chair in Genomics, Cell Biology and Aging.

\section{REFERENCES}

Aksam, E. B., Koek, A., Kiel, J. A., Jourdan, S., Veenhuis, M., and van der Klei, I. J. (2007). A peroxisomal lon protease and peroxisome degradation by autophagy play key roles in vitality of Hansenula polymorpha cells. Autophagy 3, 96-105.

Aksam, E. B., Jungwirth, H., Kohlwein, S. D., Ring, J., Madeo, F., Veenhuis, M., and van der Klei, I. J. (2008). Absence of the peroxiredoxin Pmp20 causes peroxisomal protein leakage and necrotic cell death. Free Radic. Biol. Med. 45, 1115-1124.

Aksam, E. B., de Vries, B., van der Klei, I. J., and Kiel, J. A. (2009). Preserving organelle vitality: peroxisomal quality control mechanisms in yeast. FEMS Yeast Res. 9, 808-820.

Anderson, R. M., Bitterman, K. J., Wood, J. G., Medvedik, O., and Sinclair, D. A. (2003). Nicotinamide and PNC1 govern lifespan extension by calorie restriction in Saccharomyces cerevisiae. Nature 423, 181-185.

Andrade-Navarro, M. A., SanchezPulido, L., and McBride, H. M. (2009). Mitochondrial vesicles: an ancient process providing new links to peroxisomes. Curr. Opin. Cell Biol. 21, 560-567.

Antonenkov, V. D., Grunau, S., Ohlmeier, S., and Hiltunen, J. K. (2010). Peroxisomes are oxidative organelles. Antioxid. Redox Signal. 13, 525-537.

Asakura, M., Okuno, T., and Takano, Y. (2006). Multiple contributions of peroxisomal metabolic function to fungal pathogenicity in Colletotrichum lagenarium. Appl. Environ. Microbiol. 72, 6345-6354.

Ashibe, B., Hirai, T., Higashi, K., Sekimizu, K., and Motojima, K. (2007). Dual subcellular localization in the endoplasmic reticulum and peroxisomes and a vital role in protecting against oxidative stress of fatty aldehyde dehydrogenase are achieved by alternative splicing. $J$. Biol. Chem. 282, 20763-20773.

Baker, A., Graham, I. A., Holdsworth, M., Smith, S. M., and Theodoulou, F. L. (2006). Chewing the fat: $\beta$ oxidation in signalling and development. Trends Plant Sci. 11, 124-132.

Bhadauria, V., Banniza, S., Vandenberg, A., Selvaraj, G., and Wei, Y. (2012). Peroxisomal alanine: glyoxylate aminotransferase AGT1 is indispensable for appressorium function of the rice blast pathogen, Magnaporthe oryzae. PLoS ONE 7:e36266. doi: 10.1371/journal.pone.0036266

Bitterman, K. J., Anderson, R. M., Cohen, H. Y., Latorre-Esteves, M., and Sinclair, D. A. (2002). Inhibition of silencing and accelerated aging by nicotinamide, a putative negative regulator of yeast sir2 and human SIRT1. J. Biol. Chem. 277, 45099-45107.

Bonekamp, N. A., Völkl, A., Fahimi, H. D., and Schrader, M. (2009). Reactive oxygen species and peroxisomes: struggling for balance. Biofactors 35, 346-355.

Calabrese, V., Cornelius, C., Cuzzocrea, S., Iavicoli, I., Rizzarelli, E., and Calabrese, E. J. (2011). Hormesis, cellular stress response and vitagenes as critical determinants in aging and longevity. Mol. Aspects Med. 32, 279-304.

Calabrese, V., Cornelius, C., DinkovaKostova, A. T., Iavicoli, I., Di Paola, R., Koverech, A., Cuzzocrea, S., Rizzarelli, E., and Calabrese, E. J. (2012). Cellular stress responses, hormetic phytochemicals and vitagenes in aging and longevity. Biochim. Biophys. Acta 1822, 753-783. 
Chang, J., Mast, F. D., Fagarasanu, A., Rachubinski, D. A., Eitzen, G. A., Dacks, J. B., and Rachubinski, R. A. (2009). Pex3 peroxisome biogenesis proteins function in peroxisome inheritance as class V myosin receptors. J. Cell Biol. 187, 233-246.

Chelstowska, A., and Butow, R. A. (1995). RTG genes in yeast that function in communication between mitochondria and the nucleus are also required for expression of genes encoding peroxisomal proteins. J. Biol. Chem. 270, 18141-18146.

Cho, Y. H., Yoo, S. D., and Sheen, J. (2006). Regulatory functions of nuclear hexokinase 1 complex in glucose signaling. Cell 127, 579-589.

Cohen, G. B., Rangan, V. S., Chen, B. K., Smith, S., and Baltimore, D. (2000). The human thioesterase II protein binds to a site on HIV-1 Nef critical for CD4 down-regulation. J. Biol. Chem. 275, 23097-23105.

Corpas, F. J., Barroso, J.B., and del Rio, L. A. (2001). Peroxisomes as a source of reactive oxygen species and nitric oxide signal molecules in plant cells. Trends Plant Sci. 6, 145-150.

D'Autréaux, B., and Toledano, M. B. (2007). ROS as signalling molecules: mechanisms that generate specificity in ROS homeostasis. Nat. Rev. Mol. Cell Biol. 8, 813-824.

Delille, H. K., Alves, R., and Schrader, M. (2009). Biogenesis of peroxisomes and mitochondria: linked by division. Histochem. Cell Biol. 131, 441-446.

del Río, L. A., Sandalio, L. M., Corpas, F. J., Palma, J. M., and Barroso, J. B. (2006). Reactive oxygen species and reactive nitrogen species in peroxisomes. Production, scavenging, and role in cell signaling. Plant Physiol. 141, 330-335.

Desikan, R., Mackerness, S. A.H., Hancock, J. T., and Neill, S. J. (2001). Regulation of the Arabidopsis transcriptome by oxidative stress. Plant Physiol. 127, 159-172.

Desvergne, B., and Wahli, W. (1999). Peroxisome proliferator-activated receptors: nuclear control of metabolism. Endocr. Rev. 20, 649-688.

Dixit, E., Boulant, S., Zhang, Y., Lee, A. S., Odendall, C., Shum, B., Hacohen, N., Chen, Z. J., Whelan, S. P., Fransen, M., Nibert, M. L., Superti-Furga, G., and Kagan, J. C. (2010). Peroxisomes are signaling platforms for antiviral innate immunity. Cell 141, 668-681.

Eldakak, A., Rancati, G., Rubinstein, B., Paul, P., Conaway, V., and Li,
R. (2010). Asymmetrically inherited multidrug resistance transporters are recessive determinants in cellular replicative ageing. Nat. Cell Biol. 12, 799-805.

Epstein, C. B., Waddle, J. A., Hale, W., Davé, V., Thornton, J., Macatee, T. L., Garner, H. R., and Butow, R. A. (2001). Genome-wide responses to mitochondrial dysfunction. Mol. Biol. Cell 12, 297-308.

Erjavec, N., Cvijovic, M., Klipp, E., and Nyström, T. (2008). Selective benefits of damage partitioning in unicellular systems and its effects on aging. Proc. Natl. Acad. Sci. U.S.A. 105, 18764-18769.

Erjavec, N., Larsson, L., Grantham, J., and Nyström, T. (2007). Accelerated aging and failure to segregate damaged proteins in Sir2 mutants can be suppressed by overproducing the protein aggregation-remodeling factor Hsp104p. Genes Dev. 21, 2410-2421.

Escaño, C. S., Juvvadi, P. R., Jin, F. J., Takahashi, T., Koyama, Y., Yamashita, S., Maruyama, J., and Kitamoto, K. (2009). Disruption of the Aopex11-1 gene involved in peroxisome proliferation leads to impaired Woronin body formation in Aspergillus oryzae. Eukaryot. Cell 8, 296-305.

Fagarasanu, A., Fagarasanu, M., Eitzen, G. A., Aitchison, J. D., and Rachubinski, R. A. (2006). The peroxisomal membrane protein Inp2p is the peroxisome-specific receptor for the myosin $\mathrm{V}$ motor Myo2p of Saccharomyces cerevisiae. Dev. Cell 10, 587-600.

Fagarasanu, A., Mast, F. D., Knoblach, B., Jin, Y., Brunner, M. J., Logan, M. R., Glover, J. N., Eitzen, G. A., Aitchison, J. D., Weisman, L.S., and Rachubinski, R. A. (2009). Myosindriven peroxisome partitioning in S. cerevisiae. J. Cell Biol. 186, 541-554.

Fagarasanu, A., Mast, F. D., Knoblach, B., and Rachubinski, R. A. (2010) Molecular mechanisms of organelle inheritance: lessons from peroxisomes in yeast. Nat. Rev. Mol. Cell Biol. 11, 644-654.

Farré, J. C., Manjithaya, R., Mathewson, R. D., and Subramani, S. (2008) PpAtg30 tags peroxisomes for turnover by selective autophagy. Dev. Cell 14, 365-376.

Feng, H., Ren, M., Chen, L., and Rubin, C. S. (2007). Properties, regulation and in vivo functions of a novel protein kinase D: C. elegans DKF-2 links diacylglycerol second messenger to the regulation of stress responses and lifespan. J. Biol. Chem. 282, 31273-31288.
Flores, C. L., and Gancedo, C. (2011). Unraveling moonlighting functions with yeasts. IUBMB Life 63 , 457-462.

Footitt, S., Slocombe, S. P., Larner, V., Kurup, S., Wu, Y., Larson, T., Graham, I., Baker, A., and Holdsworth, M. (2002). Control of germination and lipid mobilization by COMATOSE, the Arabidopsis homologue of human ALDP. EMBO J. 21, 2912-2922.

Freitag, J., Ast, J., and Bölker, M. (2012). Cryptic peroxisomal targeting via alternative splicing and stop codon read-through in fungi. Nature 485, 522-525.

Gancedo, C., and Flores, C. L. (2008). Moonlighting proteins in yeasts. Microbiol. Mol. Biol. Rev. 72 197-210.

Gavva, N. R., Wen, S. C., Daftari, P., Moniwa, M., Yang, Y. M., YangFeng, L. P., Seto, E., Davie, J. R., and Shen, C. K. (2002). NAPP2, a peroxisomal membrane protein, is also a transcriptional corepressor. Genomics 79, 423-431.

Gems, D., and Partridge, L. (2008). Stress-response hormesis and aging: "that which does not kill us makes us stronger". Cell Metab. 7 , 200-203.

Geuze, H. J., Murk, J. L., Stroobants, A. K., Griffith, J. M., Kleijmeer, M. J., Koster, A. J., Verkleij, A. J. Distel, B., and Tabak, H. F. (2003). Involvement of the endoplasmic reticulum in peroxisome formation. Mol. Biol. Cell 14, 2900-2907.

Ghislain, M., Talla, E., and François, J. M. (2002). Identification and functional analysis of the Saccharomyces cerevisiae nicotinamidase gene, PNC1. Yeast 19, 215-224.

Giorgio, M., Trinei, M., Migliaccio, E., and Pelicci, P. G. (2007). Hydrogen peroxide: a metabolic by-product or a common mediator of ageing signals? Nat. Rev. Mol. Cell Biol. 8 722-728.

Goh, J., Jeon, J., Kim, K. S., Park, J., Park, S. Y., and Lee, Y. H. (2011). The PEX7-mediated peroxisomal import system is required for fungal development and pathogenicity in Magnaporthe oryzae. PLoS ONE 6:e28220. doi: 10.1371/journal.pone.0028220

Goldberg, A. A., Bourque, S. D. Kyryakov, P., Gregg, C., BoukhViner, T., Beach, A., Burstein, M. T., Machkalyan, G., Richard, V., Rampersad, S., Cyr, D., Milijevic, S., and Titorenko, V. I. (2009a). Effect of calorie restriction on the metabolic history of chronologically aging yeast. Exp. Gerontol. 44, 555-571.
Goldberg, A. A., Bourque, S. D., Kyryakov, P., Boukh-Viner, T., Gregg, C., Beach, A., Burstein, M. T., Machkalyan, G., Richard, V., Rampersad, S., and Titorenko, V. I. (2009b). A novel function of lipid droplets in regulating longevity. Biochem. Soc. Trans. 37, 1050-1055.

Goodman, J. M. (2008). The gregarious lipid droplet. J. Biol. Chem. 283, 28005-28009.

Gould, S. G., Valle, D., and Raymond, G. V. (2001). "The peroxisome biogenesis disorders," in The Metabolic and Molecular Bases of Inherited Disease, eds C. R. Scriver, A. L. Beaudet, W. S. Sly, and D. Valle (New York, NY: McGraw-Hill), 3181-3217.

Haigis, M. C., and Guarente, L. P. (2006). Mammalian sirtuinsemerging roles in physiology, aging, and calorie restriction. Genes Dev. 20, 2913-2921.

Haigis, M. C., and Sinclair, D. A. (2010). Mammalian sirtuins: biological insights and disease relevance. Annu. Rev. Pathol. 5, 253-295.

Henderson, K. A., and Gottschling, D. E. (2008). A mother's sacrifice: what is she keeping for herself? Curr. Opin. Cell Biol. 20, 723-728.

Hiltunen, J. K., Mursula, A. M., Rottensteiner, H., Wierenga, R. K., Kastaniotis, A. J., and Gurvitz, A. (2003). The biochemistry of peroxisomal $\beta$-oxidation in the yeast Saccharomyces cerevisiae. FEMS Microbiol. Rev. 27, 35-64.

Hu, J., Aguirre, M., Peto, C., Alonso, J., Ecker, J., and Chory, J. (2002). A role for peroxisomes in photomorphogenesis and development of Arabidopsis. Science 297, 405-409.

Imazaki, A., Tanaka, A., Harimoto, Y., Yamamoto, M., Akimitsu, K., Park, P., and Tsuge, T. (2010). Contribution of peroxisomes to secondary metabolism and pathogenicity in the fungal plant pathogen Alternaria alternata. Eukaryot. Cell 9, 682-694.

Islinger, M., Grille, S., Fahimi, H. D., and Schrader, M. (2012). The peroxisome: an update on mysteries. Histochem. Cell Biol. 137, 547-574.

Ivashchenko, O., Van Veldhoven, P. P., Brees, C., Ho, Y. S., Terlecky, S. R., and Fransen, M. (2011). Intraperoxisomal redox balance in mammalian cells: oxidative stress and interorganellar cross-talk. Mol. Biol. Cell 22, 1440-1451.

Jazwinski, S. M. (2005a). Yeast longevity and aging - the mitochondrial connection. Mech. Ageing Dev. 126, 243-248. 
Jazwinski, S. M. (2005b). The retrograde response links metabolism with stress responses, chromatindependent gene activation, and genome stability in yeast aging. Gene 354, 22-27.

Jazwinski, S. M. (2012). The retrograde response and other pathways of interorganelle communication in yeast replicative aging. Subcell. Biochem. 57, 79-100.

Jedd, G., and Chua, N. H. (2000). A new self-assembled peroxisomal vesicle required for efficient resealing of the plasma membrane. Nat. Cell Biol. 2, 226-231.

Jedd, G. (2011). Fungal evo-devo: organelles and multicellular complexity. Trends Cell Biol. 21, 12-19.

Jeffery, C. J. (1999). Moonlighting proteins. Trends Biochem. Sci. 24, 8-11.

Jeffery, C. J. (2009). Moonlighting proteins-an update. Mol. Biosyst. 5, 345-350.

Jeffery, C. J. (2011). Proteins with neomorphic moonlighting functions in disease. IUBMB Life 63, 489-494.

Jungwirth, H., Ring, J., Mayer, T., Schauer, A., Büttner, S., Eisenberg, T., Carmona-Gutierrez, D., Kuchler, K., and Madeo, F. (2008). Loss of peroxisome function triggers necrosis. FEBS Lett. 582, 2882-2886.

Karnik, S. K., and Trelease, R. N. (2005). Arabidopsis peroxin 16 coexists at steady state in peroxisomes and endoplasmic reticulum. Plant Physiol. 138, 1967-1981.

Kersten, S., Desvergne, B., and Wahli, W. (2000). Roles of PPARs in health and disease. Nature 405, 421-424.

Kim, J. W., and Dang, C. V. (2005). Multifaceted roles of glycolytic enzymes. Trends Biochem. Sci. 30, 142-150.

Kimura, A., Takano, Y., Furusawa, I., and Okuno, T. (2001). Peroxisomal metabolic function is required for appressorium-mediated plant infection by Colletotrichum lagenarium. Plant Cell 13, 1945-1957.

Koepke, J. I., Wood, C. S., Terlecky, L. J., Walton, P. A., and Terlecky, S. R. (2008). Progeric effects of catalase inactivation in human cells. Toxicol. Appl. Pharmacol. 232, 99-108.

Kohlwein, S. D. (2010). Triacylglycerol homeostasis: insights from yeast. $J$. Biol. Chem. 285, 15663-15667.

Kos, W., Kal, A. J., van Wilpe, S., and Tabak, H. F. (1995). Expression of genes encoding peroxisomal proteins in Saccharomyces cerevisiae is regulated by different circuits of transcriptional control. Biochim. Biophys. Acta 1264, 79-86.

Kunze, M., Pracharoenwattana, I., Smith, S. M., and Hartig, A. (2006).
A central role for the peroxisomal membrane in glyoxylate cycle function. Biochim. Biophys. Acta 1763, 1441-1452.

Lai, C. Y., Jaruga, E., Borghouts, C., and Jazwinski, S. M. (2002). A mutation in the ATP2 gene abrogates the age asymmetry between mother and daughter cells of the yeast Saccharomyces cerevisiae. Genetics 162, 73-87.

Legakis, J. E., Koepke, J. I., Jedeszko, C., Barlaskar, F., Terlecky, L. J., Edwards, H. J., Walton, P. A., and Terlecky, S. R. (2002). Peroxisome senescence in human fibroblasts. Mol. Biol. Cell 13, 4243-4255.

Léon, S., Zhang, L., McDonald, W. H., Yates, J., Cregg, J. M., and Subramani, S. (2006). Dynamics of the peroxisomal import cycle of PpPex20p: ubiquitin-dependent localization and regulation. J. Cell Biol. 172, 67-78.

Li, A., Zhang, R., Pan, L., Tang, L., Zhao, G., Zhu, M., Chu, J., Sun, X., Wei, B., Zhang, X., Jia, J., and Mao, L. (2011). Transcriptome analysis of $\mathrm{H}_{2} \mathrm{O}_{2}$-treated wheat seedlings reveals a $\mathrm{H}_{2} \mathrm{O}_{2}$-responsive fatty acid desaturase gene participating in powdery mildew resistance. PLoS ONE 6:e28810. doi: 10.1371/journal.pone. 0028810

Lin, Y., Cluette-Brown, J. E., and Goodman, H. M. (2004). The peroxisome deficient Arabidopsis mutant sse 1 exhibits impaired fatty acid synthesis. Plant Physiol. 135, 814-827.

Lin, Y., Sun, L., Nguyen, L. V., Rachubinski, R. A., and Goodman, H. M. (1999). The Pex16p homolog SSE1 and storage organelle formation in Arabidopsis seeds. Science $284,328-330$.

Lin, S. J., and Sinclair, D. (2008). "Molecular mechanisms of aging: insights from budding yeast," in Molecular Biology of Aging, eds L. P. Guarente, L. Partridge, and D. C. Wallace (Cold Spring Harbor, NY: Cold Spring Harbor Laboratory Press), 483-516.

Lingard, M. J., Monroe-Augustus, M., and Bartel, B. (2009). Peroxisomeassociated matrix protein degradation in Arabidopsis. Proc. Natl. Acad. Sci. U.S.A. 106, 4561-4566.

Liu, B., Larsson, L., Caballero, A., Hao, X., Oling, D., Grantham, J., and Nyström, T. (2010). The polarisome is required for segregation and retrograde transport of protein aggregates. Cell 140, 257-267.

Liu, F., Ng, S. K., Lu, Y., Low, W., Lai, J., and Jedd, G. (2008). Making two organelles from one: Woronin body biogenesis by peroxisomal protein sorting. J. Cell Biol. 180, 325-339.

Liu, F., Lu, Y., Pieuchot, L., Dhavale, T., and Jedd, G. (2011). Import oligomers induce positive feedback to promote peroxisome differentiation and control organelle abundance. Dev. Cell 21, 457-468.

Liu, Z., and Butow, R. A. (2006) Mitochondrial retrograde signaling. Annu. Rev. Genet. 40, 159-185.

Low, C. P., Liew, L. P., Pervaiz, S., and Yang, H. (2005). Apoptosis and lipoapoptosis in the fission yeast Schizosaccharomyces pombe. FEMS Yeast Res. 5, 1199-1206.

Ma, C., Agrawal, G., and Subramani, S. (2011). Peroxisome assembly: matrix and membrane protein biogenesis. J. Cell Biol. 193, 7-16.

Ma, L., Gao, Y., Qu, L., Chen, Z., Li, J., Zhao, H., and Deng, X. W. (2002). Genomic evidence for COP1 as a repressor of light-regulated gene expression and development in Arabidopsis. Plant Cell 14, 2383-2398.

Manjithaya, R., Nazarko, T. Y., Farré, J. C., and Subramani, S. (2010). Molecular mechanism and physiological role of pexophagy. FEBS Lett. 584, 1367-1373.

Mast, F. D., Fagarasanu, A., Knoblach, B., and Rachubinski, R. A. (2010). Peroxisome biogenesis: something old, something new, something borrowed. Physiology 25, 347-356.

Mast, F. D., Li, J., Virk, M. K., Hughes, S. C., Simmonds, A. J., and Rachubinski, R. A. (2011). A Drosophila model for the Zellweger spectrum of peroxisome biogenesis disorders. Dis. Model. Mech. 4 659-672.

Mathur, J. (2009). Rapid peroxisomal responses to ROS suggest an alternative mechanistic model for postbiogenesis peroxisomal life cycle in plants. Plant Signal. Behav. 4 787-789.

Michalik, L., and Wahli, W. (2006) Involvement of PPAR nuclear receptors in tissue injury and wound repair. J. Clin. Invest. 116, 598-606.

Michalik, L., Desvergne, B., Dreyer, C. Gavillet, M., Laurini, R. N., and Wahli, W. (2002). PPAR expression and function during vertebrate development. Int. J. Dev. Biol. 46, 105-114.

Mohan, K. V., Som, I., and Atreya, C. D. (2002). Identification of a type 1 peroxisomal targeting signal in a viral protein and demonstration of its targeting to the organelle. J. Virol. $76,2543-2547$.

Morita, M., Kurochkin, I. V., Motojima, K., Goto, S., Takano, T., Okamura,
S., Sato, R., Yokota, S., and Imanaka, T. (2000). Insulin-degrading enzyme exists inside of rat liver peroxisomes and degrades oxidized proteins. Cell Struct. Funct. 25, 309-315.

Motley, A. M., Hettema, E. H., Ketting, R., Plasterk, R., and Tabak, H. F. (2000). Caenorhabditis elegans has a single pathway to target matrix proteins to peroxisomes. EMBO Rep. 1 , 40-46.

Neher, M. D., Weckbach, S., HuberLang, M. S., and Stahel, P. F. (2012). New insights into the role of peroxisome proliferator-activated receptors in regulating the inflammatory response after tissue injury. PPAR Res. 2012, 728461.

Neuspiel, M., Schauss, A. C., Braschi, E., Zunino, R., Rippstein, P., Rachubinski, R. A., AndradeNavarro, M. A., and McBride, H. M. (2008). Cargo-selected transport from the mitochondria to peroxisomes is mediated by vesicular carriers. Curr. Biol. 18, 102-108.

Nyathi, Y., and Baker, A. (2006). Plant peroxisomes as a source of signalling molecules. Biochim. Biophys. Acta 1763, 1478-1495.

Petriv, O. I., Pilgrim, D. B., Rachubinski, R. A., and Titorenko, V. I. (2002). RNA interference of peroxisome-related genes in $C$. elegans: a new model for human peroxisomal disorders. Physiol. Genomics 10, 79-91.

Poirier, Y., Antonenkov, V. D., Glumoff, T., and Hiltunen, J. K. (2006). Peroxisomal $\beta$-oxidation-a metabolic pathway with multiple functions. Biochim. Biophys. Acta 1763, 1413-1426.

Powers, J. M., and Moser, H. W. (1998). Peroxisomal disorders: genotype, phenotype, major neuropathologic lesions, and pathogenesis. Brain Pathol. 8, 101-120.

Rattan, S. I. (2008). Hormesis in aging. Ageing Res. Rev. 7, 63-78.

Rucktäschel, R., Girzalsky, W., and Erdmann, R. (2011). Protein import machineries of peroxisomes. Biochim. Biophys. Acta 1808, 892-900.

Schlüter, A., Real-Chicharro, A., Gabaldón, T., Sánchez-Jiménez, F., and Pujol, A. (2010). PeroxisomeDB 2.0, an integrative view of the global peroxisoma metabolome. Nucleic Acids Res. 38 D800-D805.

Sen, N., Hara, M. R., Kornberg, M. D., Cascio, M. B., Bae, B. I., Shahani, N., Thomas, B., Dawson, T. M., Dawson, V. L., Snyder, S. H., and Sawa, A. (2008). Nitric oxideinduced nuclear GAPDH activates 
p300/CBP and mediates apoptosis. Nat. Cell Biol. 10, 866-873.

Seo, J. G., Lai, C. Y., Miceli, M. V., and Jazwinski, S. M. (2007). A novel role of peroxin PEX6, suppression of aging defects in mitochondria. Aging Cell 6, 405-413.

Shi, Y., and Shi, Y. (2004). Metabolic enzymes and coenzymes in transcription-a direct link between metabolism and transcription? Trends Genet. 20, 445-452.

Sinclair, A. M., Trobacher, C. P., Mathur, N., Greenwood, J. S., and Mathur, J. (2009). Peroxule extension over ER-defined paths constitutes a rapid subcellular response to hydroxyl stress. Plant $J$. $59,231-242$.

Slabas, A. R., Ndimba, B., Simon, W. J., and Chivasa, S. (2004). Proteomic analysis of the Arabidopsis cell wall reveals unexpected proteins with new cellular locations. Biochem. Soc. Trans. 32, 524-528.

Spitaler, M., and Cantrell, D. A. (2004). Protein kinase $\mathrm{C}$ and beyond. Nat. Immunol. 5, 785-790.

Starai, V. J., Takahashi, H., Boeke, J. D., and Escalante-Semerena, J. C. (2003). Short-chain fatty acid activation by acyl-coenzyme A synthetases requires SIR2 protein function in Salmonella enterica and Saccharomyces cerevisiae. Genetics 163, 545-555.

Steinkraus, K. A., Kaeberlein, M., and Kennedy, B. K. (2008). Replicative aging in yeast: the means to the end. Annu. Rev. Cell Dev. Biol. 24, 29-54.
Tenney, K., Hunt, I., Sweigard, J., Pounder, J. I., McClain, C., Bowman, E. J., and Bowman, B. J. (2000). Hex-1, a gene unique to filamentous fungi, encodes the major protein of the Woronin body and functions as a plug for septal pores. Fungal Genet. Biol. 31, 205-217.

Terlecky, S. R., Koepke, J. I., and Walton, P. A. (2006). Peroxisomes and aging. Biochim. Biophys. Acta 1763, 1749-1754.

Terlecky, S. R., and Titorenko, V. I. (2009). Emergent Functions of the Peroxisome. Kerala, India: Research Signpost.

Thines, E., Weber, R. W. S., and Talbot, N. J. (2000). MAP kinase and protein kinase A-dependent mobilization of triacylglycerols and glycogen during appressorium turgor generation by Magnaporthe grisea. Plant Cell 12, 1703-1718.

Thoms, S., Grønborg, S., and Gärtner, J. (2009). Organelle interplay in peroxisomal disorders. Trends Mol. Med. 15, 293-302.

Titorenko, V. I., Ogrydziak, D. M., and Rachubinski, R. A. (1997). Four distinct secretory pathways serve protein secretion, cell surface growth, and peroxisome biogenesis in the yeast Yarrowia lipolytica. Mol. Cell. Biol. 17, 5210-5226.

Titorenko, V. I., and Rachubinski, R. A. (1998). Mutants of the yeast Yarrowia lipolytica defective in protein exit from the endoplasmic reticulum are also defective in peroxisome biogenesis. Mol. Cell. Biol. 18, 2789-2803.

Titorenko, V. I., and Rachubinski, R. A. (2004). The peroxisome: orchestrating important developmental decisions from inside the cell. J. Cell Biol. 164, 641-645.

Titorenko, V. I., and Rachubinski, R. A. (2009). Spatiotemporal dynamics of the ER-derived peroxisomal endomembrane system. Int. Rev. Cell Mol. Biol. 272, 191-244.

Titorenko, V. I., and Terlecky, S. R. (2011). Peroxisome metabolism and cellular aging. Traffic 12, 252-259.

Traven, A., Wong, J. M., Xu, D., Sopta, M., and Ingles, C. J. (2001). Interorganellar communication. Altered nuclear gene expression profiles in a yeast mitochondrial DNA mutant. J. Biol. Chem. 276 4020-4027.

Van Veldhoven, P. P. (2010). Biochemistry and genetics of inherited disorders of peroxisomal fatty acid metabolism. J. Lipid Res. 51, 2863-2895.

Veal, E. A., Day, A. M., and Morgan, B. A. (2007). Hydrogen peroxide sensing and signaling. Mol. Cell 26, $1-14$.

Wanders, R. J., and Waterham, H. R. (2006). Biochemistry of mammalian peroxisomes revisited. Annu. Rev. Biochem. 75, 295-332.

Wang, Z. Y., Jenkinson, J. M., Holcombe, L. J., Soanes, D. M., Veneault-Fourrey, C., Bhambra, G. K., and Talbot, N. J. (2005). The molecular biology of appressorium turgor generation by the rice blast fungus Magnaporthe grisea. Biochem. Soc. Trans. 33, 384-388.

Zhou, C., Slaughter, B. D., Unruh, J. R., Eldakak, A., Rubinstein, B., and Li, R. (2011). Motility and segregation of Hsp104-associated protein aggregates in budding yeast. Cell 147, 1186-1196.

Conflict of Interest Statement: The authors declare that the research was conducted in the absence of any commercial or financial relationships that could be construed as a potential conflict of interest.

Received: 24 May 2012; accepted: 28 June 2012; published online: 17 July 2012 Citation: Beach A, Burstein MT, Richard $V R$, Leonov A, Levy S and Titorenko VI (2012) Integration of peroxisomes into an endomembrane system that governs cellular aging. Front. Physio. 3:283. doi: 10.3389/fphys.2012.00283

This article was submitted to Frontiers in Integrative Physiology, a specialty of Frontiers in Physiology.

Copyright (c) 2012 Beach, Burstein, Richard, Leonov, Levy and Titorenko. This is an open-access article distributed under the terms of the Creative Commons Attribution License, which permits use, distribution and reproduction in other forums, provided the original authors and source are credited and subject to any copyright notices concerning any third-party graphics etc. 